\title{
Adaptive hybrid steepest descent algorithms involving an inertial extrapolation term for split monotone variational inclusion problems
}

\author{
Zheng Zhou ${ }^{1}$, Bing Tan ${ }^{1}$, and Songxiao $\operatorname{Li}^{1}$ \\ ${ }^{1}$ University of Electronic Science and Technology of China
}

May 28, 2021

\begin{abstract}
In this paper, we discuss the split monotone variational inclusion problem and propose two new inertial algorithms in infinitedimensional Hilbert spaces. As well as, the iterative sequence by the proposed algorithms converges strongly to the solution of a certain variational inequality with the help of the hybrid steepest descent method. Furthermore, an adaptive step size criterion is considered in suggested algorithms to avoid the difficulty of calculating the operator norm. Finally, some numerical experiments show that our algorithms are realistic and summarize the known results.
\end{abstract}

\section{Hosted file}

ZZ13-SMVIP-MMA.pdf available at https://authorea.com/users/315224/articles/524012-adaptivehybrid-steepest-descent-algorithms-involving-an-inertial-extrapolation-term-for-splitmonotone-variational-inclusion-problems

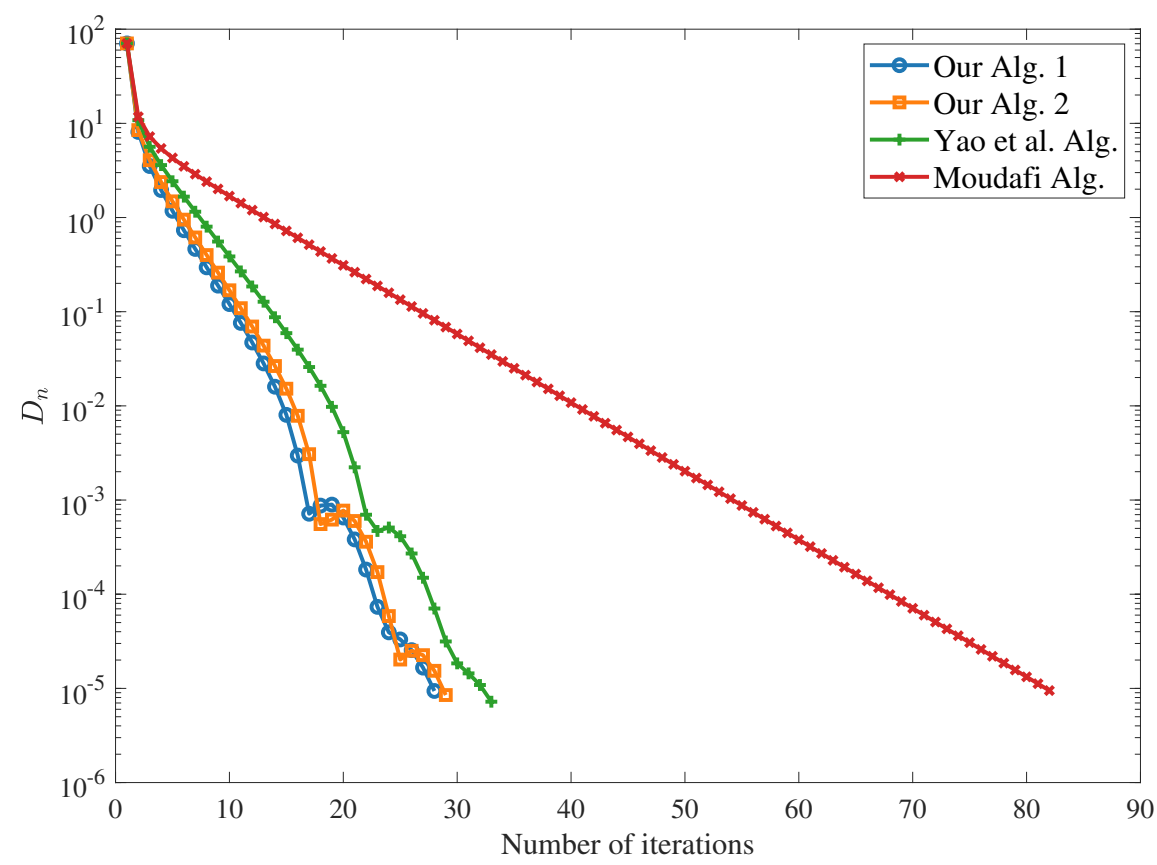



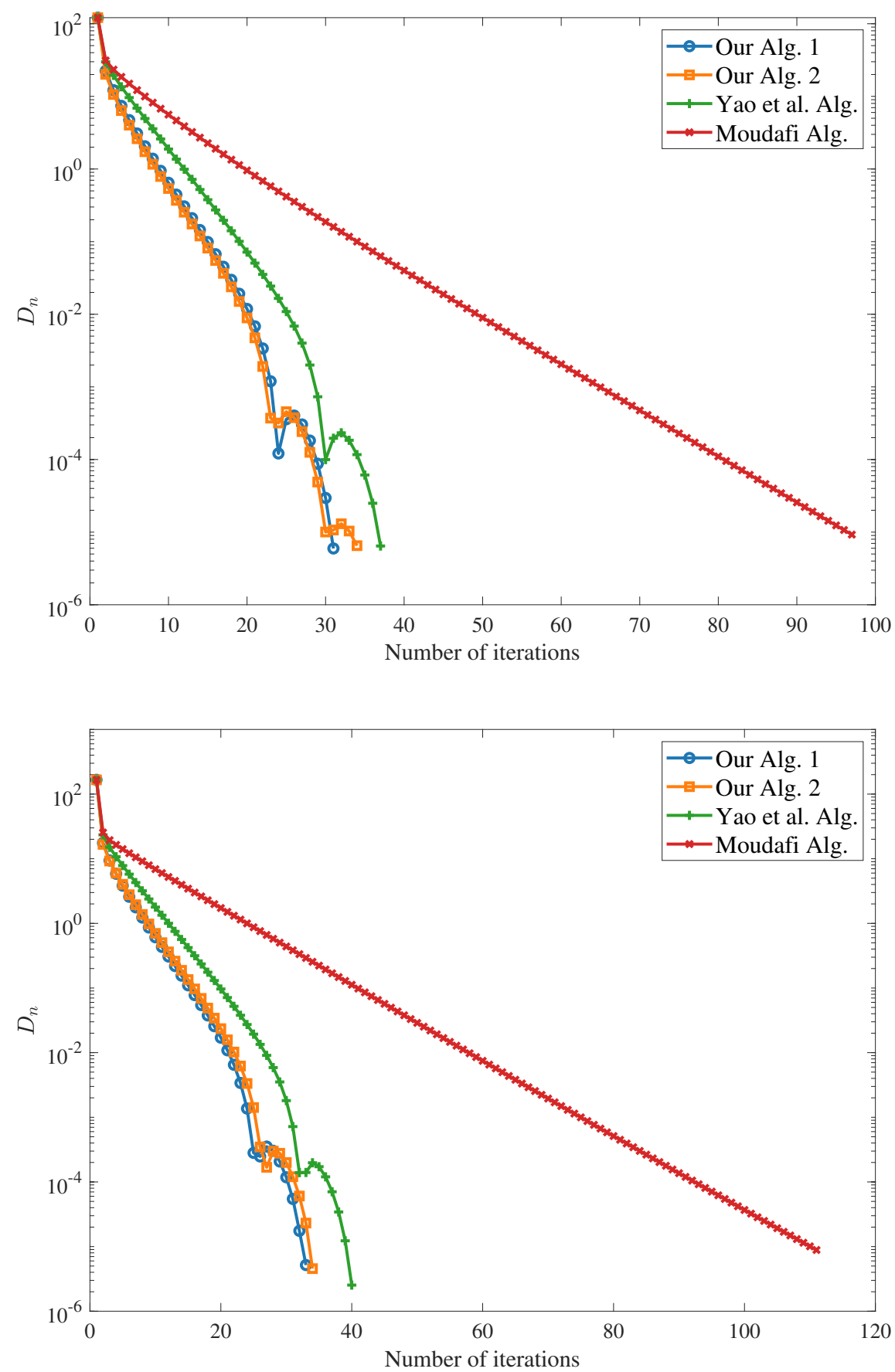

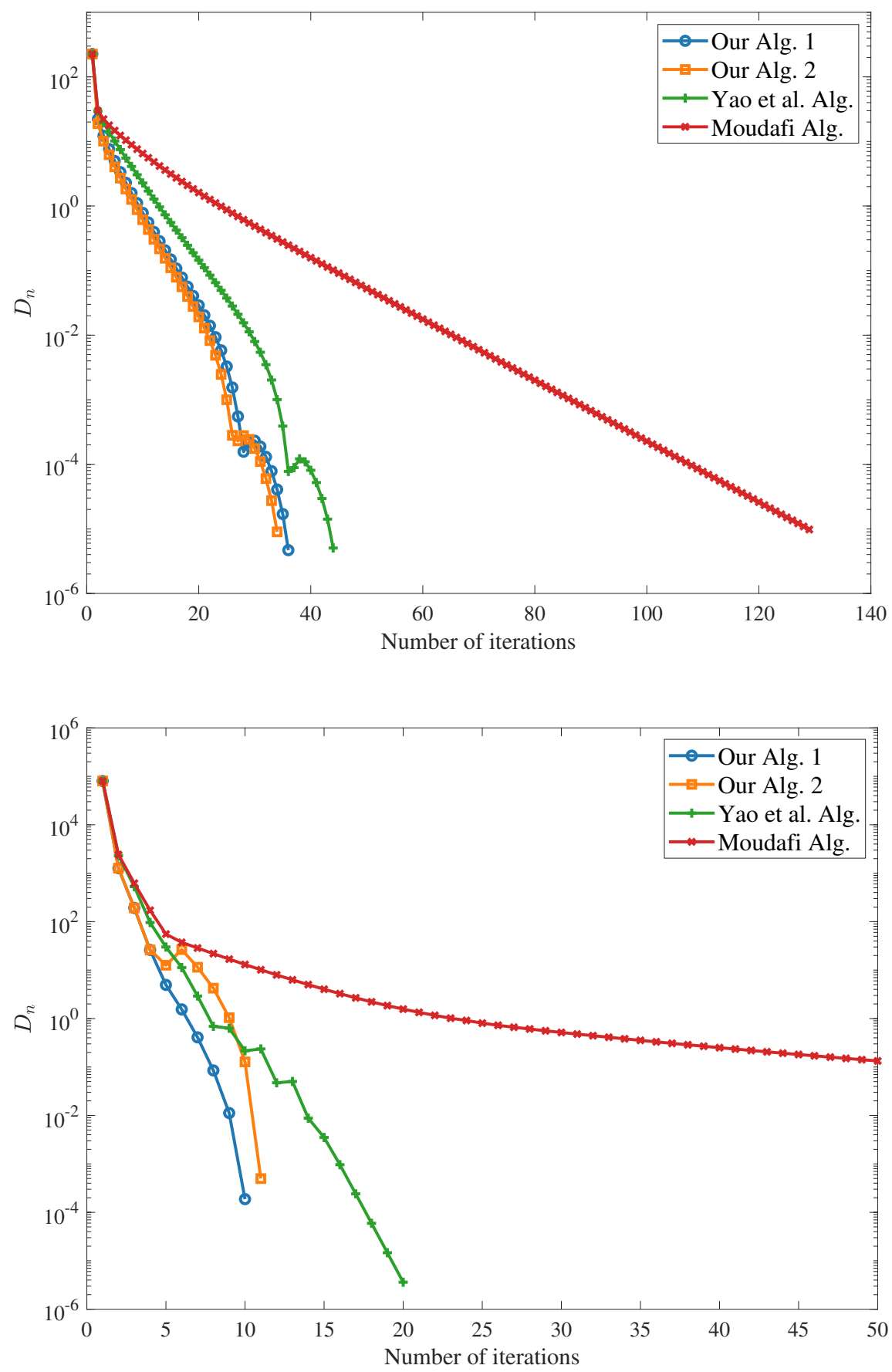

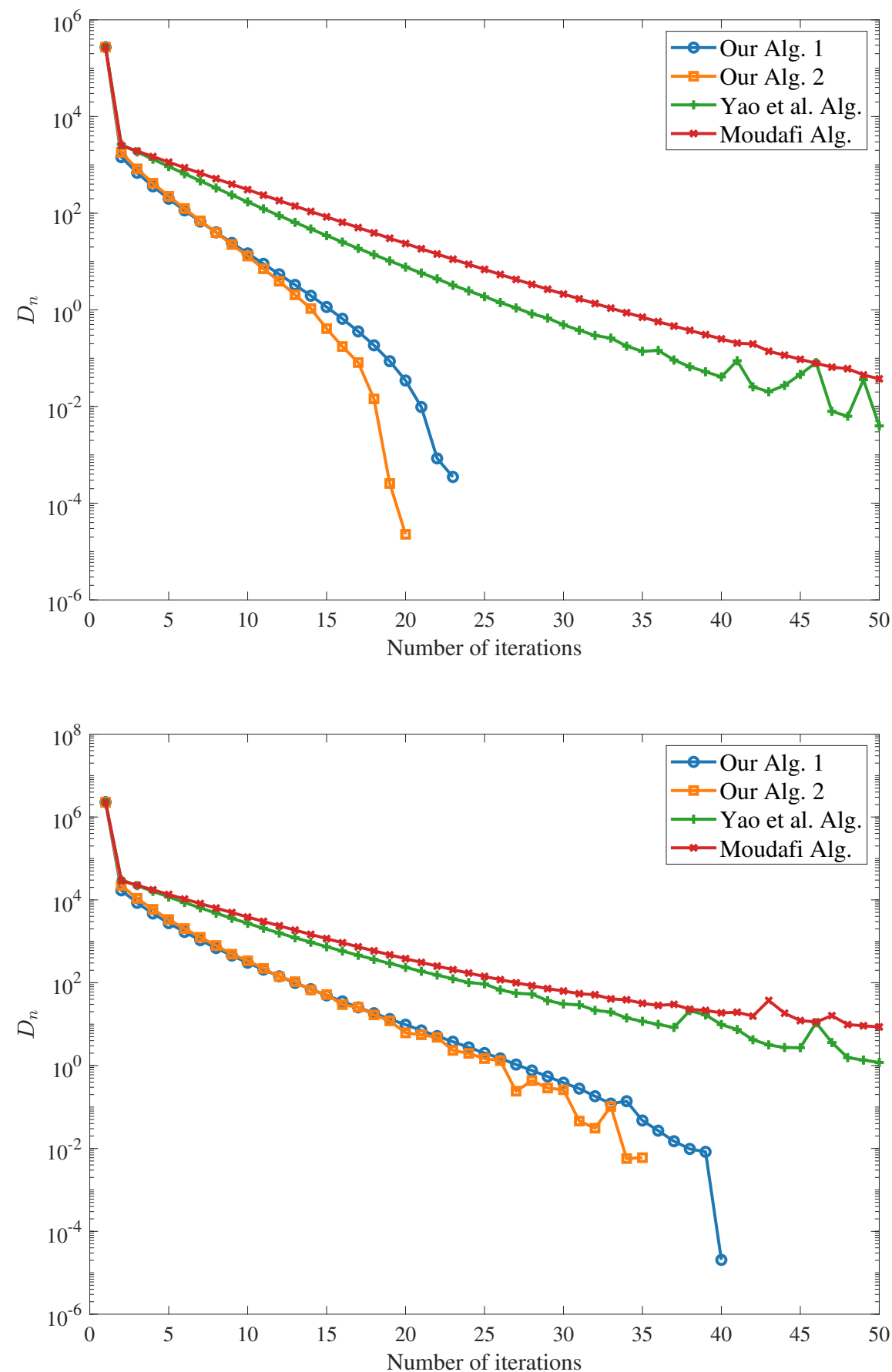

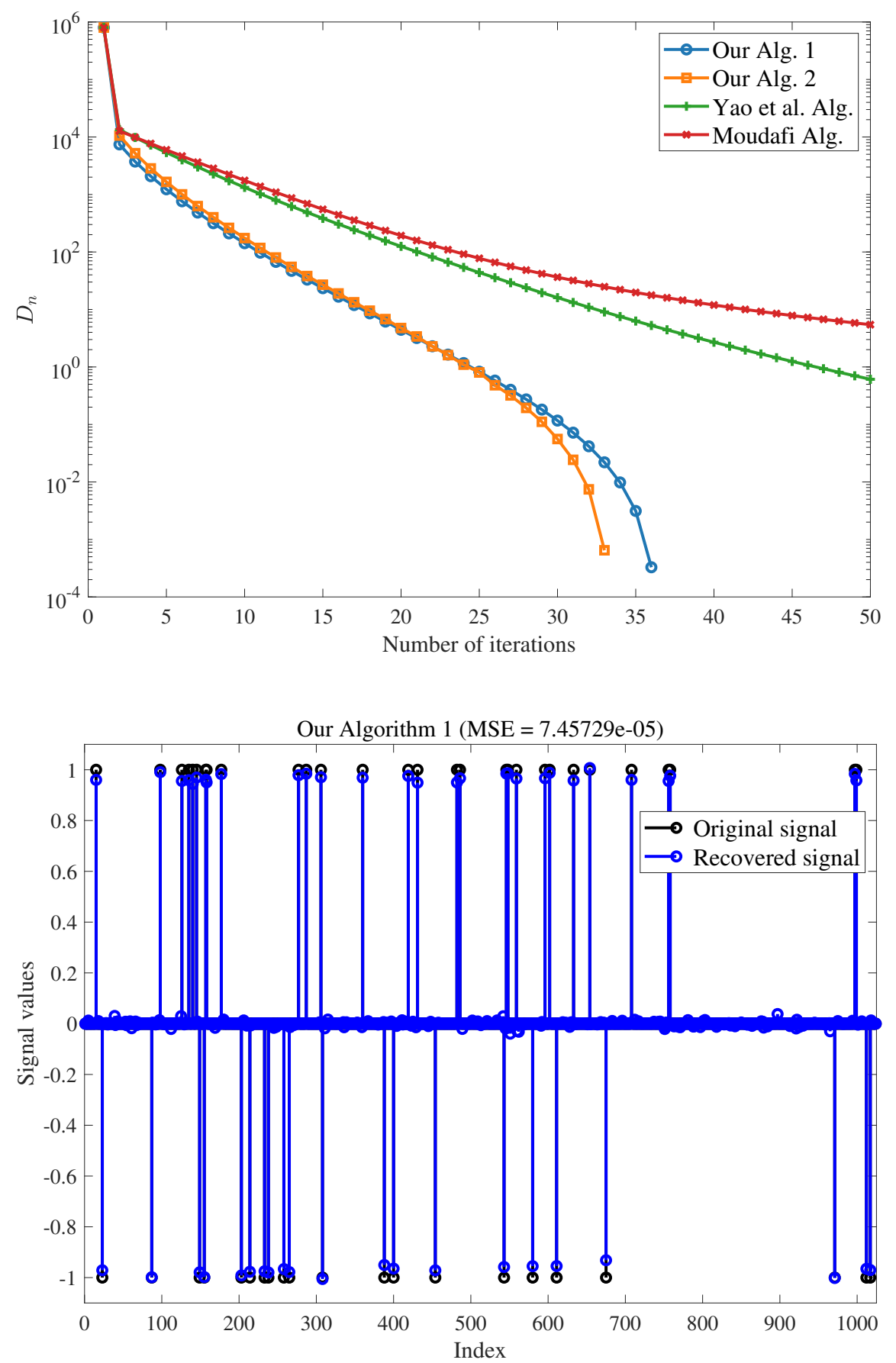


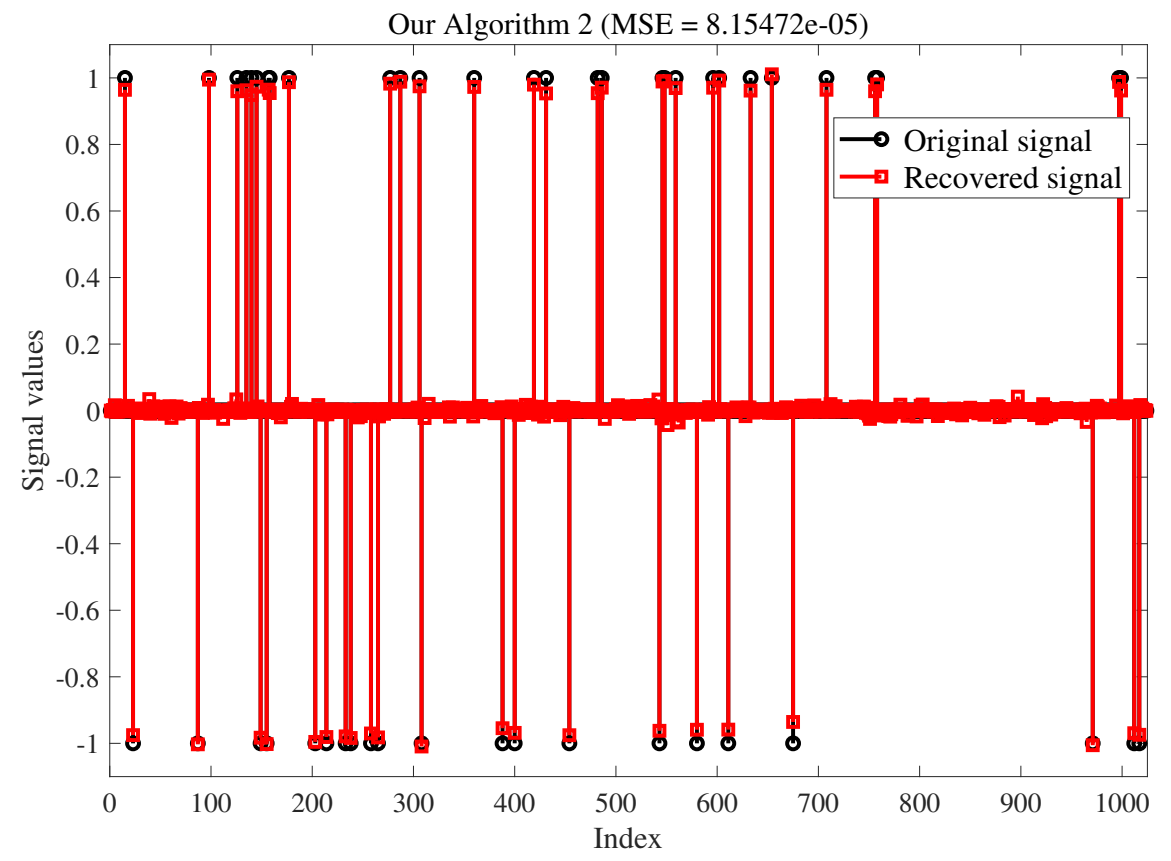

